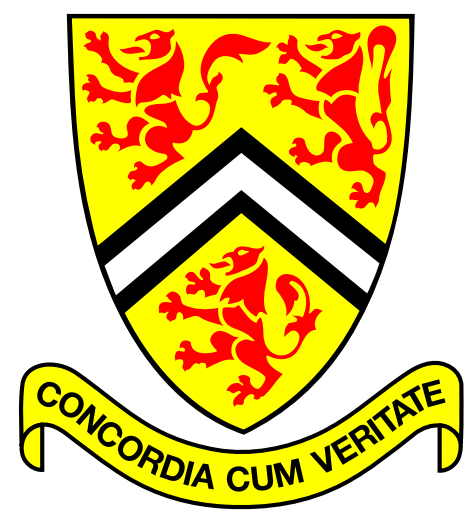

\title{
A New Method of Channel Feedback Quantization for High Data Rate MIMO Systems
}

Mehdi Ansari Sadrabadi, Amir K. Khandani, and Farshad Lahouti

Coding \& Signal Transmission Laboratory

Department of Electrical \& Computer Engineering

University of Waterloo

Waterloo, Ontario, Canada, N2L 3G1

Technical Report UW-E\&CE\#2004-05

March 20, 2004 


\title{
A New Method of Channel Feedback
}

\section{Quantization for High Data Rate MIMO}

\section{Systems}

\author{
Mehdi Ansari Sadrabadi, Amir K. Khandani and Farshad Lahouti \\ Coding \& Signal Transmission Laboratory(www.cst.uwaterloo.ca) \\ Dept. of Elec. and Comp. Eng., University of Waterloo \\ Waterloo, ON, Canada, N2L 3G1 \\ Tel: 519-884-8552, Fax: 519-888-4338 \\ e-mail: \{mehdi, khandani, farshad\}@cst.uwaterloo.ca
}

\begin{abstract}
In this work we study a Multiple-Input Multiple-Output wireless system where the channel state information is partially available at the transmitter through a feedback link. Based on Singular Value Decomposition, the MIMO channel is split into independent sub-channels which allows separate, and therefore, efficient decoding of the transmitted data signal. Effective feedback of the required spatial channel information entails efficient quantization/encoding of a Haar unitary matrix. The parameter reduction of an $n \times n$ unitary matrix to its $n^{2}-n$ basic parameters is performed through Givens decomposition. We prove that Givens matrices of a Haar unitary matrix are statistically independent. Subsequently, we derive the Probability Distribution Function (PDF) of the corresponding matrix elements. Based on these analyses, an efficient quantization scheme is proposed.
\end{abstract}


The performance evaluation is provided for a scenario where the rates allocated to each independent channel are selected according to its corresponding gain. The results indicate a significant performance improvement compared to the performance of MIMO systems without feedback at the cost of a very low-rate feedback link.

\section{Index Terms}

MIMO systems, Singular Value Decomposition, Givens matrix, Quantization, Rate Allocation

\section{INTRODUCTION}

Multiple-Input Multiple-Output (MIMO) communication systems have received considerable attention in response to the increasing requirements of high spectral efficiency in wireless communications. In fact, the capacity of MIMO systems equipped with $M_{t}$ transmit and $M_{r}$ receive antennas scales up almost linearly with the minimum of $M_{t}$ and $M_{r}$ in flat Rayleigh fading environments [1] [2].

In recent years researchers have examined the transmission strategies for MIMO systems in which the transmitter and/or the receiver have full or partial knowledge of the Channel State Information (CSI). In [3], it has been shown that the achievable bit rate when perfect CSI is available both at the transmitter and the receiver is significantly higher than that when CSI is only available at the receiver. Due to practical restrictions such as an imperfect channel estimation and a limited feedback data rate, CSI is not perfect at the transmitter. However, unlike the single antenna systems where exploiting CSI at the transmitter does not significantly enhance the capacity, in multiple antenna systems the capacity is substantially improved through even partial CSI [4].

When CSI is available at the transmitter of a Multiple-Input Single-Output (MISO) system, beam-forming can be used to exploit transmit diversity through spatial match filtering. In the context of MISO systems, several quantization schemes have been suggested to feed back instantaneous CSI to the transmitter. A simple and effective scheme has been 
suggested for a $3 \mathrm{G}$ wireless standard [5]. In [6] the authors design a codebook of beamformer vectors with the objective of minimizing the outage probability. Similar works, titled in single-sub-stream precoding, have been reported in [7-9], where the codebook design criterion is derived to maximize the received Signal to Noise Ratio (SNR).

For MIMO systems, the problem of quantizing CSI is more involved than for MISO systems. In [10] a precoder is combined with a space-time encoder. The precoder is designed to reduce an upper bound on the worst pairwise codeword error probability conditioned on imperfect CSI at the transmitter. In [11], by assuming the availability of partial CSI at the transmitter of a MIMO system, a criterion has been presented to design a precoder based on the capacity maximization. However, [11] has not provided a practical approach to design such a precoder when the number of receive antennas is more than one. In [12], the achievable rate of a MIMO fading channel with the quantized spatial information of the channel at the transmitter is studied. It determines the minimum feedback rate needed to achieve the capacity associated with the perfect CSI at the transmitter. However, in [12], a practical approach to quantize the spatial information of the channel is not presented. In this paper we present a technique to address the need for a practical feedback scheme for a MIMO system (as opposed to a MISO).

We consider the situation in which a MIMO channel is split into several independent sub-channels by means of Singular Value Decomposition (SVD) based on the CSI at the transmitter and the receiver. This allows independent decoding of the sub-channels and results in a low decoding complexity. In general, the optimum Maximum Likelihood (ML) decoding in a MIMO system without feedback is equivalent to a lattice decoding problem which incurs significant complexity. Lower complexity decoding algorithms can be devised by the proper design of a transmit strategy, e.g., the Bell Labs Layered Space Time system [1]. However, this is achieved at the cost of degraded performance [13] [14]. This indicates that, in addition to the gain in the SNR performance, a reduction in the decoding complexity is another important advantage of a closed loop MIMO system based on the SVD.

In this work the modulation format is selected to match the sub-channel SNR on each sub-channel. In this scheme the spatial information of the channel and the constellation 
index of each sub-channel is needed at the transmitter. We develop an algorithm to quantize the spatial information of the channel based on minimizing the interference between the sub-channels. The rate allocation strategy is determined at the receiver and fed back to the transmitter by using an efficient low rate approach.

The system model is described in Section II. In Section III, the parameterization and statistics of the right singular matrix of a Gaussian matrix is discussed. The unitary matrices are decomposed into Givens matrices and the statistical properties of Givens matrices are theoretically derived. The feedback design is developed according to these properties and the decoding strategy at the receiver. In Section IV, a feedback scheme for transferring the rate information of each sub-channel is discussed. In Section V the simulation results are presented. Section VI concludes the paper.

\section{SYSTEM MODEL}

We consider an independent and identically distributed block fading channel model. For a multiple transmit antenna system with $M_{t}$ transmit and $M_{r}$ receive antennas, the model leads to the following complex baseband representation of the received signal:

$$
\mathbf{y}=\mathbf{H x}+\mathbf{n},
$$

where $\mathbf{x}$ is the $M_{t} \times 1$ vector of the transmitted symbols, $\mathbf{H}$ is the $M_{r} \times M_{t}$ channel matrix, $\mathbf{n}$ is the $M_{r} \times 1$ zero mean Gaussian noise vector with the autocorrelation $\sigma 2 \mathbf{I}$ where $\mathbf{I}$ is the identity matrix, and $\mathbf{y}$ is the received signal. The power constraint of the transmitted signal is defined as $E\left(\mathbf{x x}^{*}\right)=\mathcal{E} \mathbf{I}$, where $E$ represents the expectation and (.)* is the hermitian of (.). The elements of the channel matrix $\mathbf{H}$ are circularly symmetric complex Gaussian distributed with zero mean and unit variance.

The SVD of matrix $\mathbf{H}$ is defined as [15]

$$
\mathbf{H}=\mathbf{V} \boldsymbol{\Lambda} \mathbf{U}^{*},
$$

where $\mathbf{V}$ and $\mathbf{U}$ are the unitary matrices, and $\boldsymbol{\Lambda}$ is a diagonal matrix. If $\mathbf{U}$ is available at the transmitter and the transmitted signal is pre-filtered by $\mathbf{U}$, then the received signal is given 
by

$$
\begin{aligned}
\mathbf{y} & =\mathbf{H U} \mathbf{x}+\mathbf{n} \\
& =\mathbf{V} \boldsymbol{\Lambda} x+\mathbf{n} .
\end{aligned}
$$

The receiver filters the received vector $\mathbf{y}$ by $\mathbf{V}^{*}$,

$$
\begin{aligned}
& \mathbf{r}=\mathbf{V}^{*} \mathbf{y} \\
& =\Lambda x+\mathbf{n} .
\end{aligned}
$$

Therefore, a MIMO channel with $M_{t}$ transmit antennas and $M_{r}$ receive antennas is transformed to 'rank $\mathbf{H}$ ' parallel sub-channels. This transformation substantially reduces the decoding complexity. In the transition from (3) to (4), we take advantage of the fact that the elements of $\mathbf{n}$ are statistically independent, and rotating $\mathbf{n}$ by the unitary matrix $\mathbf{V}^{*}$ does not change the distribution of the noise.

As it can be seen in (4), the sub-channels provide different gains corresponding to $\boldsymbol{\Lambda}$. We consider a case in which data is transmitted and received separately in each subchannel with different rates and with equal energy. It can be shown that the use of equal energy maximizes the rate under the assumption of continuous approximation for a cubical shaping region (subject to a constraint on total energy). Noting that the proposed scheme uses an orthonormal precoder, it is easy to see that this condition translates to equal energy per transmit antenna (balanced power amplifiers). This method involves the allocation of an appropriate data rate to each sub-channel, while a certain target error rate on each subchannel is met. By this assumption, the transmitter requires the rate information of each sub-channel in addition to the right singular matrix of the channel.

\section{Feedback Design: Channel Singular Matrix Quantization}

In the scenario described above, the transmitter needs to know the right singular matrix of the channel. We assume that a noiseless feedback link from the receiver to the transmitter is available. By the SVD of $\mathbf{H}$ at the receiver, the unitary matrix $\mathbf{U}$ is computed, quantized and sent to the transmitter. 
If we assume the quantization error $\Delta U$ for $\mathbf{U}$, the received signal is

$$
\mathbf{r}=\Lambda x+\Lambda U^{*} \Delta U x+\mathbf{n} .
$$

The quantization scheme is based on minimizing the interference between the parallel subchannels since the receiver strategy is to detect the data in each sub-channel independently. The variance of the interference signal is expressed as follows:

$$
\begin{aligned}
E\left(\left\|\boldsymbol{\Lambda} \mathbf{U}^{*} \boldsymbol{\Delta} U x\right\|^{2}\right) & =E \operatorname{Tr}\left(\boldsymbol{\Lambda} \mathbf{U}^{*} \Delta U x \mathbf{x}^{*} \Delta U^{*} \mathbf{U} \boldsymbol{\Lambda}\right) \\
& =\lambda E \operatorname{Tr}\left(\mathbf{U}^{*} \boldsymbol{\Delta} U x \mathbf{x}^{*} \boldsymbol{\Delta} U^{*} \mathbf{U}\right) \\
& =\lambda E \operatorname{Tr}\left(\boldsymbol{\Delta} U \boldsymbol{\Delta} U^{*} \mathbf{x} \mathbf{x}^{*}\right) \\
& =\lambda \mathcal{E} E \operatorname{Tr}\left(\|\boldsymbol{\Delta} U\|^{2}\right)
\end{aligned}
$$

where $E\left(\Lambda^{2}\right)=\lambda \mathbf{I}, E\left(\mathbf{x x}^{*}\right)=\mathcal{E} \mathbf{I}$ and $\operatorname{Tr}$ denotes the trace function. In (6), we use the property that the singular values of a Gaussian matrix are independent from the corresponding singular vectors [16], and also the equality $\operatorname{Tr}(\mathbf{A B})=\operatorname{Tr}(\mathbf{B A})$. As a result, minimizing the mean of the interference power leads to the minimization of the Frobenius norm of $\Delta \mathbf{U}$. In order to minimize the interference, the unitary matrix $\mathbf{U}$ should be quantized, based on minimizing the expression in (6). In the following, we examine the statistical properties of the underlying unitary matrices.

\section{A. Statistics of Singular Matrices of a Random Gaussian Matrix}

In most analytic studies of MIMO systems, the channel between the transmitter and the receiver is assumed to be Rayleigh fading. This indicates that the entries of the channel matrix are statistically independent and identically distributed and have a complex Gaussian distribution with a zero mean. We are interested in the probability distribution of the singular matrices ${ }^{1}$ of the mentioned channel matrix in the space of $M(n)$, namely the group of $n \times n$ unitary matrices. It is known that such a random unitary matrix takes its values uniformly from $M(n)$ in the sense of the following property [17]:

\footnotetext{
${ }^{1}$ The probability distribution of a matrix is the joint PDF of its elements.
} 
Theorem 1 Let us assume that $\boldsymbol{U}$ is a singular matrix of a random Gaussian matrix. For all $\boldsymbol{V} \in M(n)$, the distribution of $\boldsymbol{U}$ and $\boldsymbol{V} \boldsymbol{U}$ are the same.

Such a distribution is called the Haar distribution and the corresponding unitary matrices are called Haar unitary matrices [17]. We refer to this property as the right invariance property. Also, the left invariance property can be easily derived from Theorem 1 .

A complex $n \times n$ matrix can be described by $2 n 2$ real parameters. However, the definition of a unitary matrix implies there is a dependency between these parameters. The number of equations describing this dependency for an $n \times n$ unitary matrix is $n+2\left(\begin{array}{l}n \\ 2\end{array}\right)$ (as the norm of each column is unit and every two columns are orthogonal to each other). Therefore, a unitary matrix $\mathbf{U}$ has $n 2=2 n 2-\left(n+2\left(\begin{array}{l}n \\ 2\end{array}\right)\right)$ independent parameters. Here, for the purpose of matrix decomposition using SVD, $n$ out of $n 2$ parameters are also redundant since SVD can be performed such that the diagonal elements of $\mathbf{U}$ in (2) are set to be real. Several different approaches such as the Cayley transform, Householder reflection, and Givens rotations can be used to parameterize a complex $n \times n$ unitary matrix $\mathbf{U}$ in its $n 2-n$ real parameters [15].

In this work we consider the matrix decomposition using Givens matrices. Besides their ability to decompose the unitary matrix to the minimum number of parameters, the resulting parameters are statistically independent (Theorem 2). The independence property facilitates the quantization procedure.

A complex unitary matrix $\mathbf{U}$ can be decomposed in terms of the products of Givens matrices [15], i.e.,

$$
\mathbf{U}=\prod_{k=1}^{n-1} \prod_{i=k+1}^{n} \mathbf{G}(k, i)
$$

where each $\mathbf{G}(k, i)$ is an $n \times n$ unitary matrix with two parameters, $c$, and, $s$ given as follows: 


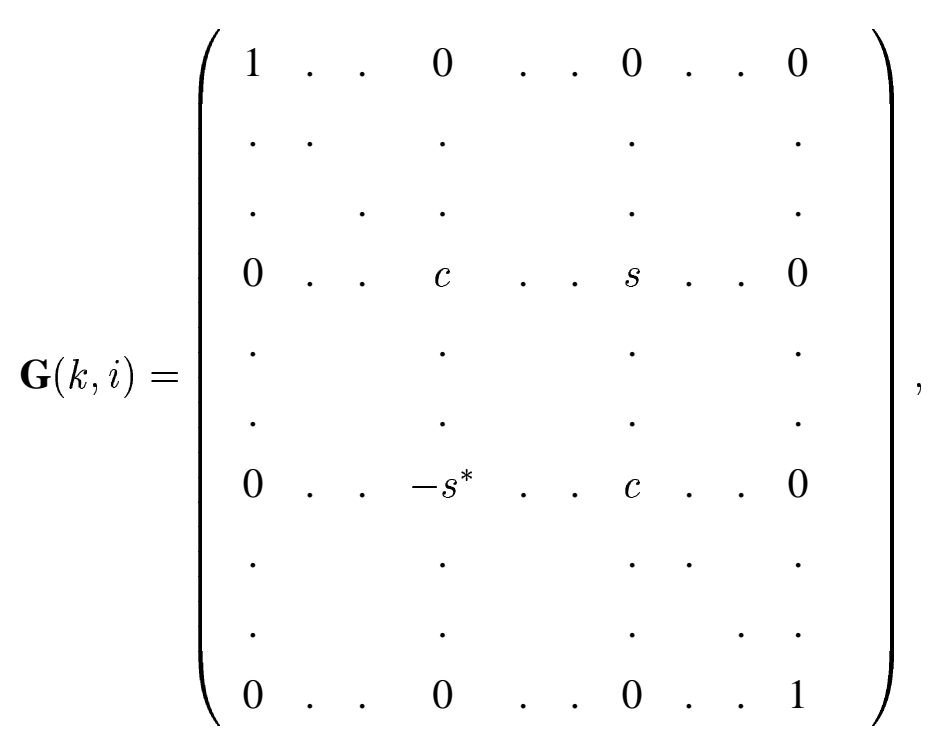

where $c$ is in the position $(k, k)$ and $(i, i), s$ is in $(k, i)$ and $-s^{*}$ is in $(i, k), k<i$. The other diagonal elements of the matrix $\mathbf{G}(k, i)$ are 1 and the remaining elements are zero. Since $\mathbf{G}(k, i)$ is a unitary matrix, then $|c| 2+|s| 2=1$. In this work we can assume that $c$ is real since the SVD operation allows $\mathbf{U}$ to be multiplied by an arbitrary diagonal unitary matrix (See Appendix A for more details).

In the following, the statistical properties of Givens matrices corresponding to a Haar unitary matrix $\mathbf{U}$ will be derived. This will be used later to determine the quantization strategy. The key point of the codebook design for a Haar unitary matrix is the following result.

Theorem 2 Let us assume that $\boldsymbol{U}$ is an $n \times n$ unitary matrix with a Haar distribution which is decomposed into Givens matrices as in (7). The set of Givens matrices $\{\boldsymbol{G}(k, i)\}$ for $1 \leq k<i \leq n$ are statistically independent of each other. Moreover, the PDF of the elements of $\boldsymbol{G}(k, i)$ is

$$
\begin{array}{r}
p_{k, i}(c, \angle s)=p_{k, i}(c) p(\angle s)=\frac{i-k}{\pi} c^{2(i-k)-1}, \\
0 \leq c \leq 1, \quad \angle s \in[-\pi, \pi] .
\end{array}
$$

Proof: See Appendix A. 


\section{B. Quantization of Unitary Matrices}

Based on the criterion presented for the quantizer design in (6), the distortion measure of the quantizer is defined as follows:

$$
\begin{aligned}
D(U) & =\frac{1}{2} E \operatorname{Tr}(\|\mathbf{U}-\widehat{\mathbf{U}}\| 2) \\
& =\frac{1}{2} E \operatorname{Tr}\left((\mathbf{U}-\widehat{\mathbf{U}})\left(\mathbf{U}^{*}-\widehat{\mathbf{U}}^{*}\right)\right) \\
& =E \operatorname{Tr}\left(\mathbf{I}-\mathcal{R}\left(\widehat{\mathbf{U}} \mathbf{U}^{*}\right)\right),
\end{aligned}
$$

where $\mathcal{R}($.$) is the real part of (.). Substituting (7) in (10), we derive the first order approxi-$ mation of $D(U)$ as follows:

$$
\begin{aligned}
D(U) & =E \operatorname{Tr}\left(\mathbf{I}-\mathcal{R}\left(\prod_{l=1}^{n-1} \prod_{j=l+1}^{n} \widehat{\mathbf{G}}(l, j)\left(\prod_{l=1}^{n-1} \prod_{j=l+1}^{n} \mathbf{G}(l, j)\right)^{*}\right)\right) \\
& =E \operatorname{Tr}\left(\mathbf{I}-\mathcal{R}\left(\prod_{l=1}^{n-1} \prod_{j=l+1}^{n}(\widehat{\mathbf{G}}(l, j)-\mathbf{G}(l, j)+\mathbf{G}(l, j))\left(\prod_{l=1}^{n-1} \prod_{j=l+1}^{n} \mathbf{G}(l, j)\right)^{*}\right)\right) \\
& \simeq E \sum_{k=1}^{n-1} \sum_{i=k+1}^{n} \operatorname{Tr} \mathcal{R}\left(\prod_{l=1}^{k} \prod_{j=l+1}^{i-1} \mathbf{G}(l, j)\right. \\
& \left.(\mathbf{G}(k, i)-\widehat{\mathbf{G}}(k, i)) \prod_{l=k}^{n-1} \prod_{j=i+1}^{n} \mathbf{G}(l, j)\left(\prod_{l=1}^{n-1} \prod_{j=l+1}^{n} \mathbf{G}(l, j)\right)^{*}\right) \\
& =E \operatorname{Tr}\left(\mathcal{R}\left(\sum_{k=1}^{n-1} \sum_{i=k+1}^{n}(\mathbf{G}(k, i)-\widehat{\mathbf{G}}(k, i)) \mathbf{G}^{*}(k, i)\right)\right) \\
& =E \operatorname{Tr}\left(\sum_{k=1}^{n-1} \sum_{i=k+1}^{n}\left(\mathbf{I}-\mathcal{R}\left(\widehat{\mathbf{G}}(k, i) \mathbf{G}^{*}(k, i)\right)\right)\right) \\
& \left.=\sum_{k=1}^{n-1} \sum_{i=k+1}^{n} D_{(G, i}(k, i)\right),
\end{aligned}
$$

where $D(G)$ is defined as follows:

$$
D(G)=\frac{1}{2} E \operatorname{Tr}(\|\mathbf{G}-\widehat{\mathbf{G}}\| 2),
$$

and $\widehat{\mathbf{G}}$ is the quantized version of $\mathbf{G}$. In the derivation of (11), we have applied the fact that $\operatorname{Tr}(\mathbf{A B})=\operatorname{Tr}(\mathbf{B A})$ and ignored the terms containing the multipliers $(\mathbf{G}(k, i)-\widehat{\mathbf{G}}(k, i)), 1 \leq$ 
$k<i \leq n$ of order two or higher. In the following, we use

$$
\mathbf{G}=\left(\begin{array}{cc}
c & s \\
-s^{*} & c
\end{array}\right)
$$

to refer to the non-trivial part of (8).

1) Method A: The basic parameters of the Givens matrix in (8), named $c$ and $\theta=\angle s$, are quantized as $\widehat{c}$ and $\widehat{\theta}$, independently. The transmitter uses $\widehat{c}$ and $\widehat{\theta}$ to construct $\widehat{\mathbf{G}}$ as follows:

$$
\widehat{\mathbf{G}}=\left(\begin{array}{cc}
\widehat{c} & |\widehat{s}| e^{j \widehat{\theta}} \\
-|\widehat{s}| e^{-j \widehat{\theta}} & \widehat{c}
\end{array}\right)
$$

where $|\widehat{s}|=\sqrt{1-\widehat{c}^{2}}$. According to the construction scheme in (14), $\widehat{\mathbf{G}}$ is also unitary. It can be easily demonstrated (Appendix B) that the first order approximation of $\|\Delta \mathbf{G}\| 2$ is

$$
\begin{aligned}
\|\Delta \mathbf{G}\|^{2} & =\|\mathbf{G}-\widehat{\mathbf{G}}\| 2 \\
& \simeq \frac{2}{1-c 2}(c-\widehat{c}) 2+2\left(1-c^{2}\right)(\theta-\widehat{\theta}) 2 .
\end{aligned}
$$

By applying (15), the distortion defined in (12), is

$$
D(G) \simeq E\left(\frac{(c-\widehat{c}) 2}{1-c 2}\right)+E\left(1-c^{2}\right) E(\theta-\widehat{\theta}) 2
$$

By applying (11) and (15), we write

$$
D(U) \simeq \sum_{k=1}^{n-1} \sum_{i=k+1}^{n} E\left(\frac{\left(c_{k, i}-\widehat{c}_{k, i}\right)^{2}}{1-c_{k, i}^{2}}\right)+E\left(1-c_{k, i}^{2}\right) E\left(\theta_{k, i}-\widehat{\theta}_{k, i}\right) 2,
$$

where $c_{k, i}$ and $\theta_{k, i}$ are the parameters of $\mathbf{G}(k, i)$, and $\widehat{c}_{k, i}$ and $\widehat{\theta}_{k, i}$ are the parameters of $\widehat{\mathbf{G}}_{k, i}$. We apply (9) to simplify the following expression,

$$
E\left(1-c_{k, i}^{2}\right)=\frac{1}{2(i-k)+1}
$$

By substituting (18) in (17), we have

$$
D(U) \simeq \sum_{k=1}^{n-1} \sum_{i=k+1}^{n} E\left(\frac{\left(c_{k, i}-\widehat{c}_{k, i}\right) 2}{1-c_{k, i}^{2}}\right)+\frac{1}{2(i-k)+1} E\left(\theta_{k, i}-\widehat{\theta}_{k, i}\right)^{2} .
$$


We design Linde-Buzo-Gray (LBG) quantizers for different $c_{k, i}$ and $\theta_{k, i}$ to minimize

$$
E\left(\frac{\left(c_{k, i}-\widehat{c}_{k, i}\right) 2}{1-c_{k, i}^{2}}\right)
$$

and,

$$
E\left(\theta_{k, i}-\widehat{\theta}_{k, i}\right) 2
$$

respectively. The quantizer for $\theta$ follows the conventional approach to iterative design of a scalar LBG quantizer [18], while for parameter $c$ the iterative design procedure should use the following reconstruction value,

$$
\widehat{c}=\frac{E\left(\frac{c}{1-c 2}\right)}{E\left(\frac{1}{1-c 2}\right)},
$$

which is easily derived by setting $\frac{\partial}{\partial \widehat{c}} E\left(\frac{(c-\widehat{c}) 2}{1-c 2}\right)=0$.

We utilize dynamic programming to find the optimum allocation of bits among the quantizers. We use a trellis diagram with $B+1$ states and $n 2-n$ stages to allocate $B$ bits to the quantizers of the independent parameters $c_{k, i}$ and $\theta_{k, i}, 1 \leq i<k \leq n$ of the $n \times n$ unitary matrix. The $l$ th state in $j$ th stage corresponds to the distortion caused by the $j$ th parameter using $l-1$ bits. In the trellis diagram, each branch represents the difference between the number of bits corresponding to the two ending states on the branch. The search through the trellis determines the path with minimum overall distortion and the corresponding number of bits for each parameter.

2) Method B: In this method we quantize each Givens matrix as a unit and define a new parameterization for this purpose. The non-trivial part of a Givens matrix can be shown as follows:

$$
\mathbf{G}=\left(\begin{array}{cc}
\cos (\eta) & e^{j \theta} \sin (\eta) \\
-e^{-j \theta} \sin (\eta) & \cos (\eta)
\end{array}\right)
$$

where $0 \leq \theta \leq 2 \pi$ and $0 \leq \eta \leq \pi$. According to (12), the distortion for $\mathbf{G}$, relative to a reference matrix with parameters $\eta_{0}$ and $\theta_{0}$, is

$$
D_{0}(G)=1-E\left(\cos (\eta) \cos \left(\eta_{0}\right)+\sin (\eta) \sin \left(\eta_{0}\right) \cos \left(\theta-\theta_{0}\right)\right)
$$


We use the LBG algorithm to determine the regions and centroids of the two-dimensional quantizers corresponding to various $(\eta, \theta)$. The distortion function is

$$
D=\sum_{m=1}^{M} \int_{R_{m}} D_{m}(G) p(\eta, \theta) d \eta d \theta,
$$

where $R_{m}$ is the $m$ th quantization region and $M$ is the number of quantization partitions. The centroid $\left(\eta_{m}, \theta_{m}\right)$ is determined iteratively by minimizing the distortion function in the region $R_{m}$ (see Appendix $\mathrm{C}$ ),

$$
\begin{gathered}
\theta_{m}=\tan ^{-1}\left(\frac{\varsigma_{m}}{\gamma_{m}}\right) \\
\eta_{m}=\tan ^{-1}\left(\frac{\sqrt{\varsigma_{m} 2+\gamma_{m} 2}}{\int_{R_{m}} \cos ^{l+1}(\eta) \sin (\eta) d \eta d \theta}\right),
\end{gathered}
$$

where

$$
\gamma_{m}=\int_{R_{m}} \cos ^{l}(\eta) \sin 2(\eta) \cos (\theta) d \eta d \theta
$$

and

$$
\varsigma_{m}=\int_{R_{m}} \cos ^{l}(\eta) \sin 2(\eta) \sin (\theta) d \eta d \theta
$$

and $l=2(i-k)-1$, in the case of quantizing $\mathbf{G}(k, i)$ in (7). By applying the above algorithm, we design codebooks of the matrices for different rates. In this method, a trellis diagram with the same structure as the method A trellis diagram is used for optimum bit allocation. The trellis diagram contains $\frac{n 2-n}{2}$ stages, each corresponding to a Givens component of an $n \times n$ unitary matrix, and $B+1$ states ( $B$ is the number of bits). The $l$ th state in $j$ th stage corresponds to the distortion caused by the $j$ th Givens matrix using $l-1$ bits.

\section{Feedback Design:Encoding of Rate Allocation Information}

Besides the quantized right singular matrix of the channel that is fed back to the transmitter, information pertaining the rate that will be allocated to each sub-channel is also fed back. This indicates a set of $M_{t}$ indices from a set of $N_{R}$ predetermined rates, e.g., the different modulation schemes. Obviously, the total rate is bounded, and since we 
can perform the SVD of the channel matrix so that the singular values become ordered, the $M_{t}$ indices correspond to an ordered set of increasing positive integers (rates). To encode this information, we can use a trellis diagram with $N_{R}$ states and $M_{t}$ stages (Figure 1). The states correspond to the set of possible rates in an increasing fashion and there is a branch from each state to another state in the next stage only if the entering state is located at the same or at a lower level position. Each path in the trellis then corresponds to a set of sub-channel rates whose index is chosen by the receiver and fed back to the transmitter. The trellis structure exploits the ordering property of the rates, and therefore, allows their efficient coding at a rate of [19]

$$
R_{\text {rate }}=\left\lceil\log _{2}\left(\begin{array}{c}
M_{t}+N_{R}-1 \\
N_{R}-1
\end{array}\right)\right] .
$$

The complexity of this algorithm is very low and is, in fact, proportional to the number of states. Similar structures have been used to address the points of a block-based trellis quantizer in [19] or a pyramid vector quantizer in [20]. Also, this method of enumeration is similar in spirit to the earlier works of [21] and [22] for addressing the points of a signal constellation (shell mapping).

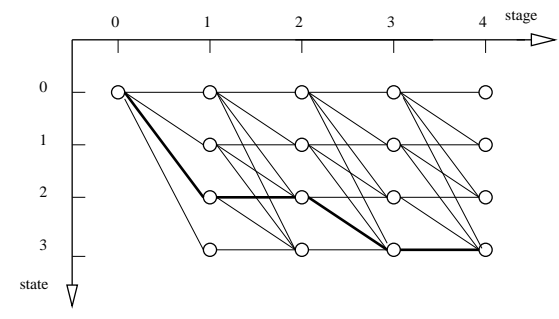

Fig. 1. An example of a trellis structure; In this example, the number of stages is 4 and the number of states is 3 .

\section{Performance Evaluation}

In this section, we present the performance results of the system described in Section II. We assume that the precoding is performed by the quantized version of the right singular matrix of the channel by applying the quantization methods presented in Section III-B. For the different sub-channels, we use different modulation schemes. The process of selecting the appropriate modulation scheme for each sub-channel is accomplished 
at the receiver. As already mentioned, we design the system to transmit data with the power $\frac{\mathcal{E}}{M_{t}}$ on each transmit antenna. At the receiver, the channel state information and the instantaneous quantization noise power is assumed to be available. For each sub-channel the probability of error is computed for different modulation schemes. The receiver selects a modulation scheme for each sub-channel that achieves the target Bit Error Rate (BER) of the system and sends the indices of the corresponding modulation schemes to the transmitter through the feedback channel that was described in Section IV. The received SNR at the $k$ th sub-channel is,

$$
S N R_{k}=\frac{\mathcal{E} \lambda_{k}^{2}}{M_{t}\left(\sigma 2+\widehat{\sigma}_{k}^{2}\right)}
$$

where $\widehat{\sigma}_{k} 2$ is the corresponding quantization noise variance of the $k$ th sub-channel. We consider a set of QAM modulation formats. At the receiver, the rate $r_{k}$ of $k$ th sub-channel is computed as follows,

$$
\max _{P\left(S N R_{k}\right) \leq P_{b}} r_{k},
$$

where $P_{b}$ is the target BER of the system and $P(S N R)$, the BER function of the modulation scheme with rate $r$, is [23]

$$
P(S N R) \approx \frac{4}{r} Q\left(\sqrt{\frac{3 r S N R}{2^{r}-1}}\right),
$$

where $Q(x)=\frac{1}{\sqrt{2 \pi}} \int_{x}^{\infty} e^{-\frac{x 2}{2}} d x$.

The SVD and Givens decomposition are performed at the receiver. The number of computations required by the SVD and Givens decomposition for an $n \times n$ matrix are $21 n 3$ and $3 n 2(n-1)$ flops, respectively [15].

Figure 2 shows the average bit rate versus SNR for different MIMO systems with $M_{t}=2$ and $M_{r}=2$ at the target $\mathrm{BER}=5 \times 10^{-3}$. We use 8 bits for the feedback of the right singular matrix. The modulation schemes we use are QAMs with bit rates between 1 and 7, inclusively, and then $N_{R}=8$. We use 6 bits for the feedback of the rate allocation vector in each transmission block (the number of bits is derived by applying (28)). The two quantization methods presented in Section III-B are compared. Method B outperforms method $\mathrm{A}$ at the cost of complexity. The average bit rate of a $2 \times 2 \mathrm{MIMO}$ system with 


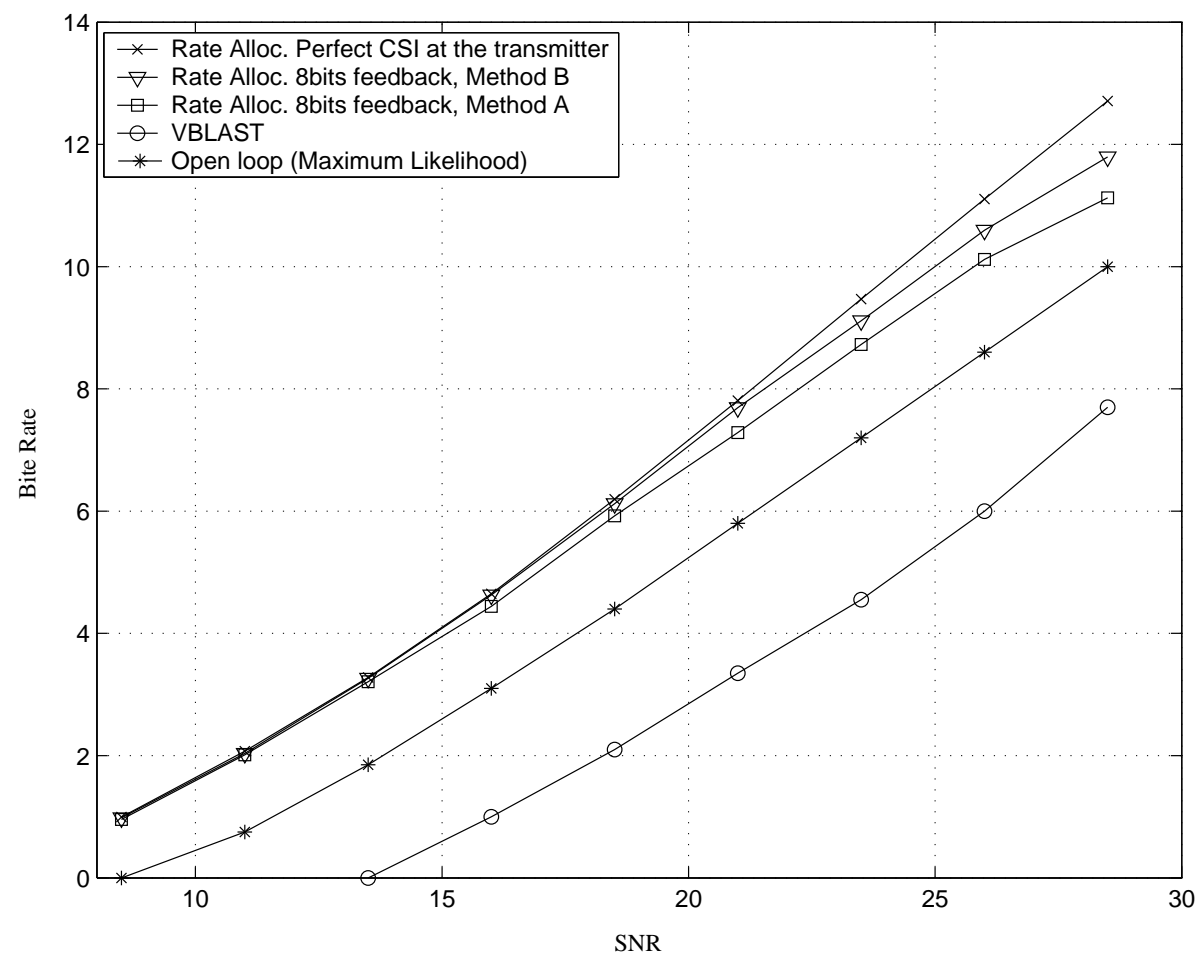

Fig. 2. The average bit rate for different schemes where $M_{t}=2$ and $M_{r}=2$. The target $\mathrm{BER}=5 \times 10^{-3}$.

ML decoding is depicted. It can be seen that the performance gain, compared to the gain of the ML decoding of the open loop system, is noticeable. For example, at the bit rate $=10$ the system has a $4 \mathrm{~dB}$ improvement in comparison to the optimum open loop system. We also compare the performance of this system with that of a V-BLAST system which is proposed as a solution to overcome the complexity problem. Figure 2 displays a significant improvement in comparison to the V-BLAST at the cost of the feedback. The performance of the system, if perfect channel information is available at the transmitter, is also depicted. Figure 3 shows the average bit rate versus SNR for different MIMO systems with $M_{t}=3$ and $M_{r}=3$ at the target $\mathrm{BER}=5 \times 10^{-3}$.

\section{CONCLUSION}

In this work we have presented efficient methods for the channel information quantization in a high data rate MIMO system. We have developed efficient algorithms for the 


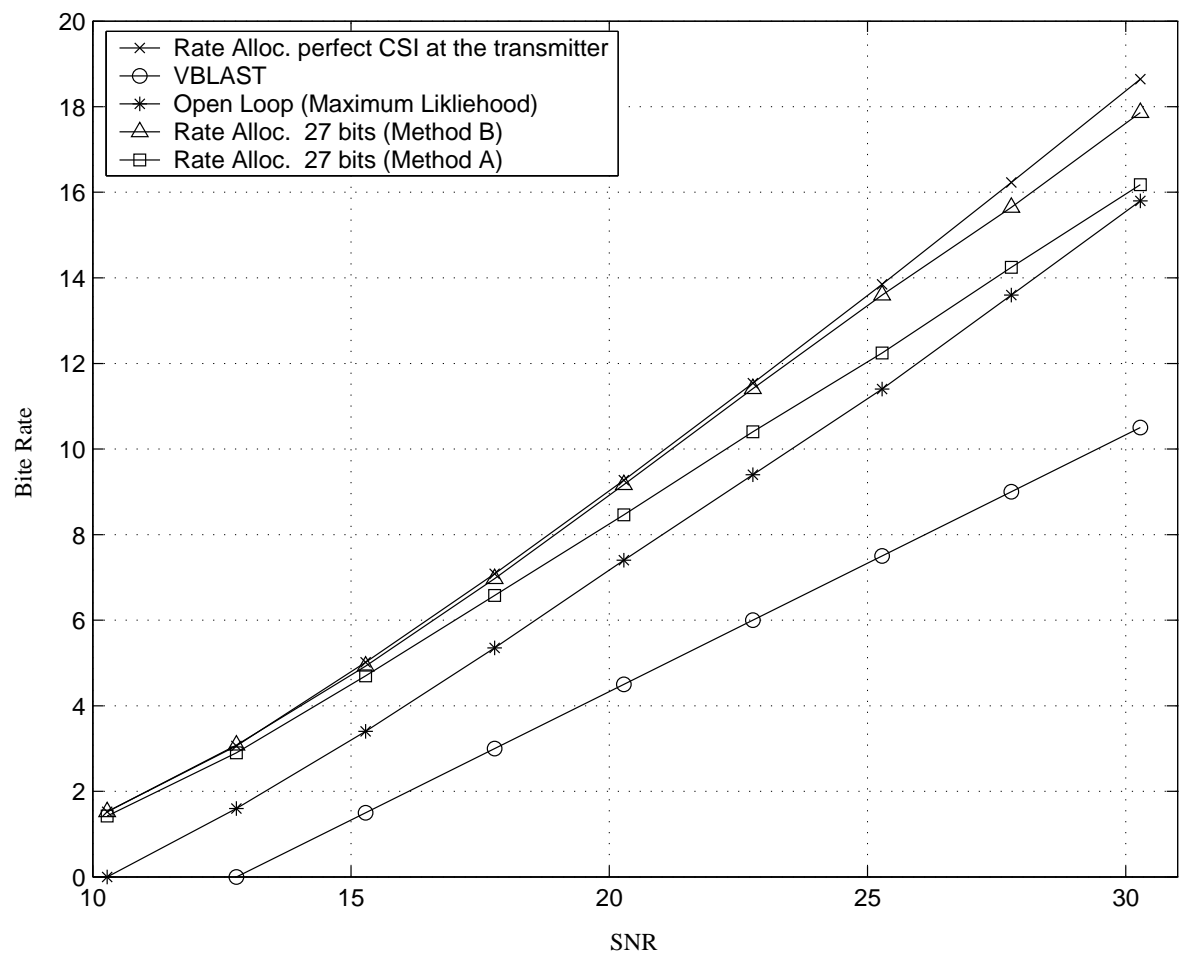

Fig. 3. The average bit rate for different schemes where $M_{t}=3$ and $M_{r}=3$. The target $\mathrm{BER}=5 \times 10^{-3}$.

quantization of the underlying unitary matrices. Also, we have presented a low rate indexing of rate allocation information. The simulation results show a significant improvement compared to MIMO systems without feedback at the cost of a very low-rate feedback link.

\section{APPENDIX A}

It is a simple matter to zero a specified entry in a vector by using a Givens matrix. Based on this fact, there is an iterative algorithm to find the Givens matrices of a unitary matrix. In each iteration step, a Givens matrix is multiplied by the resulting matrix of the last step to zero a specified entry of the product matrix. The process of decomposing $n \times n$ unitary matrix $\mathbf{U}$ into its first $n-1$ Givens component can be formulated as follows [15]:

$$
\begin{aligned}
& \mathbf{Q}^{0}=\mathbf{U} \\
& \mathbf{Q}^{i}=\mathbf{G}^{*}(1, i+1) \mathbf{Q}^{i-1}, \quad 1 \leq i \leq n-1
\end{aligned}
$$


where the superscript represents the iteration number. The elements of $\mathbf{G}(1, i+1)$ are determined such that $q_{i 1}^{i}=0,1 \leq i \leq n-1$, where $q_{k l}^{i}$ is the corresponding $(k, l)$ element of $\mathbf{Q}^{i}$. Therefore, the first column of $\mathbf{Q}^{n-1}$ is zero except for the first element. We signify the parameters of $\mathbf{G}(1, i+1)$, shown in (8) by $c_{i}$ and $s_{i}$, which can be easily computed regarding the procedure in (32) as follows:

$$
c_{i}=\frac{\sqrt{\sum_{l=1}^{i}\left|u_{l 1}\right| 2}}{\sqrt{\sum_{l=1}^{i+1}\left|u_{l 1}\right| 2}}
$$

and

$$
s_{i}=\frac{-e^{-j \angle u_{11}} u_{(i+1) 1}}{\sqrt{\sum_{l=1}^{i+1}\left|u_{l 1}\right| 2}}
$$

where $u_{k l}=\left|u_{k l}\right| e^{j \angle u_{k l}}$ is the corresponding $(k, l)$ element of $\mathbf{U}$. This process can be repeated for the following columns of the matrix $\mathbf{Q}^{n-1}$ until it becomes a diagonal matrix $\mathbf{D}_{n}$. Note that in this decomposition, $c$ in each Givens matrix is real. Suppose $\mathbf{U}$ is decomposed to its Givens components based on the above algorithm such that $\mathbf{U}=\prod_{k=1}^{n-1} \prod_{i=k+1}^{n} \mathbf{G}(k, i) \mathbf{D}_{n}$. Then, the SVD of the channel matrix defined in (2) can be written as follows:

$$
\begin{aligned}
\mathbf{H} & =\mathbf{V} \boldsymbol{\Lambda}\left(\prod_{k=1}^{n-1} \prod_{i=k+1}^{n} \mathbf{G}(k, i) \mathbf{D}_{n}\right)^{*} \\
& =\left(\mathbf{V} \mathbf{D}_{n}^{*}\right) \boldsymbol{\Lambda}\left(\prod_{k=1}^{n-1} \prod_{i=k+1}^{n} \mathbf{G}(k, i)\right)^{*} .
\end{aligned}
$$

Note that diagonal matrices $\Lambda$ and $\mathbf{D}_{n}$ are commutative and $\mathbf{V} \mathbf{D}_{n}^{*}$ is a unitary matrix. It can be inferred from (35) that the set of Givens matrices with the format we have introduced provide enough information to represent the required spatial information of the channel at the transmitter.

By using the right invariance property, the joint probability density $p\left(u_{11}, u_{21}, \ldots, u_{n 1}\right)$ of the elements of the first column of $\mathbf{U}$ is [17]

$$
p\left(u_{11}, u_{21}, \ldots, u_{n 1}\right)=\frac{2 \pi^{n / 2}}{\Gamma\left(\frac{1}{2} n\right)} \delta\left(1-\sum_{l=1}^{n}\left|u_{l 1}\right| 2\right)
$$


where $\delta($.$) is a Kronecker delta function. By integrating (36) over the variables u_{(k+1) 1}, \ldots, u_{n 1}$, we find the following expression for the joint probability density of elements $u_{11}, u_{21}, \ldots, u_{k 1}$ :

$$
p\left(u_{11}, u_{21}, \ldots, u_{k 1}\right)=K\left(1-\sum_{l=1}^{k}\left|u_{l 1}\right| 2\right)^{n-k-1}, \quad k<n
$$

where $K$ is a constant. The joint probability density of the absolute values of the elements of the first column of $\mathbf{U}$ can be easily derived as follows:

$$
p\left(\left|u_{11}\right|,\left|u_{21}\right|, \ldots,\left|u_{k 1}\right|\right)=(2 \pi)^{k} K\left(1-\sum_{l=1}^{k}\left|u_{l 1}\right| 2\right)^{n-k-1} \prod_{l=1}^{k}\left|u_{l 1}\right| .
$$

The marginal distribution of $c_{i}$ in (33) can be calculated from the joint distribution of $\left\{\left|u_{l 1}\right|\right\}_{l=1}^{i+1}$ using (38),

$$
p\left(c_{i}\right)=2 i c_{i}^{2 i-1} . \quad 0 \leq c_{i} \leq 1
$$

The joint distribution of $\left\{c_{l}\right\}_{l=1}^{i}$ is computed by using (33) and (38),

$$
p\left(c_{1}, \ldots, c_{i}\right)=\prod_{l=1}^{i}\left(2 l c_{l}^{2 l-1}\right) .
$$

The comparison of the joint distribution and the marginal distribution of $\left\{c_{i}\right\}_{i=1}^{n-1}$ in (40) and (39), respectively, implies that $\left\{c_{i}\right\}_{i=1}^{n-1}$ are statistically independent of each other.

In order to parameterize a Givens matrix in the format we stated, it is only necessary to have $c_{i}$ and the angle of complex $s_{i}$ (note that $c_{i} 2+\left|s_{i}\right| 2=1$ ). The probability distribution of the angles of the elements of a column of the Haar unitary matrix is uniformly distributed and independent [16]. Considering this argument and (34), we have

$$
p\left(\angle s_{i}\right)=\frac{1}{2 \pi} . \quad-\pi \leq \angle s_{i} \leq \pi
$$

After $n-1$ step of the decomposition process, the first column of $\mathbf{Q}^{n-1}$, defined in (32), has one non-zero element, and $\mathbf{Q}^{n-1}$ is in the following format:

$$
\mathbf{Q}^{n-1}=\left(\begin{array}{cc}
e^{j \angle u_{11}} & \mathbf{0} \\
\mathbf{0} & \mathbf{V}
\end{array}\right)
$$


where $\mathbf{V}$ is an $(n-1) \times(n-1)$ unitary matrix. In the following, we prove that $\mathbf{V}$ and the set $\{\mathbf{G}(1, i)\}_{i=2}^{n}$ are statistically independent. Since matrices $\{\mathbf{G}(1, i)\}_{i=2}^{n}$ are derived from the first column of $\mathbf{U}$, namely $\mathbf{u}_{1}$, it is sufficient to show that $\mathbf{V}$ and $\mathbf{u}_{1}$ are independent.

It is easy to show that the set of unitary matrices with a fixed first column in $M(n)$ and the group $M(n-1)$ are bijective. To show this, consider a given $n \times n$ unitary matrix $\mathbf{A}$ with a fixed first column $\mathbf{a}_{1}$, and an $n \times n$ unitary matrix $\mathbf{W}=\operatorname{diag}(1, \mathbf{Y})$, where $\mathbf{Y}$ is an arbitrary $(n-1) \times(n-1)$ unitary matrix. Using $\mathbf{B}=\mathbf{A W}$ and noting that $\mathbf{A}$ is invertible, we conclude that there exists a one-to-one correspondence between $\mathbf{B}$ and $\mathbf{W}$. On the other hand, according to Theorem 1, the probability distribution of $\mathbf{A}$ and $\mathbf{A W}$ are the same.

Noting the above arguments, we conclude that the probability density of $\mathbf{U}$ conditioned on $\mathbf{u}_{1}$ is distributed uniformly in $M(n-1)$. This means the probability density of $\mathbf{V}$ in (42) conditioned on $\mathbf{u}_{1}$ is distributed uniformly in $M(n-1)$ and, therefore, is statistically independent of $\mathbf{u}_{1}$.

Similarly, the decomposition algorithm described in (32) is applied on $\mathbf{V}$ and all the statistical arguments about $\mathbf{U}$ can be extended to $\mathbf{V}$.

\section{APPENDIX B}

In this part, the first order approximation of $\|\Delta \mathbf{G}\|^{2}$ defined in (15) is derived.

$$
\begin{array}{r}
\|\Delta \mathbf{G}\|^{2}=\|\mathbf{G}-\widehat{\mathbf{G}}\| 2 \\
=2(c-\widehat{c}) 2+2\left|\sqrt{1-c^{2}} e^{j \theta}-\sqrt{1-\widehat{c}^{2}} e^{j \widehat{\theta}}\right|^{2} \\
=2(c-\widehat{c}) 2+2\left|\sqrt{1-c^{2}} e^{j \theta}-\sqrt{1-c^{2}} e^{j \widehat{\theta}}+\sqrt{1-c^{2}} e^{j \widehat{\theta}}-\sqrt{1-\widehat{c}^{2}} e^{j \widehat{\theta}}\right|^{2} \\
=2(c-\widehat{c}) 2+2\left(1-c^{2}\right)\left|e^{j \theta}-e^{j \widehat{\theta}}\right|^{2}+2\left|e^{j \widehat{\theta}}\right|^{2}\left|\sqrt{1-c^{2}}-\sqrt{1-\widehat{c}^{2}}\right|^{2} \\
+4 \mathcal{R}\left(\left(e^{j(\theta-\widehat{\theta})}-1\right) \sqrt{1-c^{2}}\left(\sqrt{1-c^{2}}-\sqrt{1-\widehat{c}^{2}}\right)\right) \\
\simeq 2(c-\widehat{c}) 2+2\left(1-c^{2}\right)(\theta-\widehat{\theta}) 2+\frac{2 c 2}{1-c 2}(c-\widehat{c}) 2 \\
+2(\theta-\widehat{\theta}) 2 \sqrt{1-c^{2}}\left(\sqrt{1-c^{2}}-\sqrt{1-\widehat{c}^{2}}\right) \\
\simeq 2(c-\widehat{c}) 2+2\left(1-c^{2}\right)(\theta-\widehat{\theta}) 2+\frac{2 c 2}{1-c 2}(c-\widehat{c}) 2 \\
=\frac{2}{1-c 2}(c-\widehat{c}) 2+2\left(1-c^{2}\right)(\theta-\widehat{\theta}) 2 .
\end{array}
$$




\section{APPENDIX C}

The joint probability of the parameters $\eta$ and $\theta$ of the Givens matrix $\mathbf{G}(k, i)$ can be easily derived regarding to the parameterization in (21) and using (9) as follows:

$$
p(\eta, \theta)=\frac{i-k}{\pi} \sin (\eta) \cos (\eta)^{2(i-k)-1}
$$

We find the centroid $\left(\eta_{m}, \theta_{m}\right)$ in $m$ th region for the LBG quantizer in Section III-B.2, which is the minimizing point of the following function:

$$
\begin{array}{r}
D_{R_{m}}=\int_{R_{m}} D_{m}(G) p(\eta, \theta) d \eta d \theta \\
=\int_{R_{m}}\left(1-\left(\cos (\eta) \cos \left(\eta_{m}\right)+\sin (\eta) \sin \left(\eta_{m}\right) \cos \left(\theta-\theta_{m}\right)\right)\right) p(\eta, \theta) d \eta d \theta
\end{array}
$$

We find the centroid $\left(\eta_{m}, \theta_{m}\right)$ by forcing the partial derivatives of $D_{R_{m}}$ to zero. The partial derivative of $D_{R_{m}}$ respect to $\theta_{m}$ is,

$$
\frac{\partial}{\partial \theta_{m}} D_{R_{m}}=\sin \left(\eta_{m}\right) \int_{R_{m}}\left(\sin \left(\theta_{m}\right) \cos (\theta)-\cos \left(\theta_{m}\right) \sin (\theta)\right) \sin (\eta) p(\eta, \theta) d \eta d \theta
$$

By setting (46) to zero, we have,

$$
\tan \left(\theta_{m}\right)=\frac{\int_{R_{m}} \sin (\eta) \sin (\theta) p(\eta, \theta) d \eta d \theta}{\int_{R_{m}} \sin (\eta) \cos (\theta) p(\eta, \theta) d \eta d \theta}
$$

The partial derivative of $D_{R_{m}}$ respect to $\eta_{m}$ is,

$$
\frac{\partial}{\partial \eta_{m}} D_{R_{m}}=\int_{R_{m}}\left(\cos (\eta) \sin \left(\eta_{m}\right)-\sin (\eta) \cos \left(\eta_{m}\right) \cos \left(\theta-\theta_{m}\right)\right) p(\eta, \theta) d \eta d \theta .
$$

Similarly, we set (48) to zero to find $\eta_{m}$. Therefore, we have,

$$
\tan \left(\eta_{m}\right)=\frac{\cos \left(\theta_{m}\right) \int_{R_{m}} \sin (\eta) \cos (\theta) p(\eta, \theta) d \eta d \theta+\sin \left(\theta_{m}\right) \int_{R_{m}} \sin (\eta) \sin (\theta) p(\eta, \theta) d \eta d \theta}{\int_{R_{m}} \cos (\eta) p(\eta, \theta) d \eta d \theta}
$$

By using the results in (44), (47) and (48), (25) can be easily derived. 


\section{REFERENCES}

[1] G. J. Foschini and M. J. Gans, "On the limits of of wireless communications in a fading environment," Wireless Pres. Commun., vol. 6, pp. 315-335, Nov. 1998.

[2] E. Telatar, "Capacity of multi-antenna gaussian channels," Bell Labs Journal, vol. 10, Nov/Dec 1999.

[3] E. Biglieri, G. Caire, and G. Taricco, "Limiting performance of block fading channels with multiple antennas," IEEE Trans. on Information Theory, vol. 47, pp. 1273-1289, May 2001.

[4] E. Vistosky and U. Madhow, "Space-Time Transmit Precoding with Imperfect Feedback," IEEE Trans. Inform. Theory, vol. 47, pp. 2632-2639, September 2001.

[5] 3GPP Technical Specification, Group Radio Access Network, "Physical layer procedures (FDD)," vol. 5.6.0, Sept. 2003. TS 25.214.

[6] K. K. Mukkavilli, A. Sabharwal, E. Erkipand, and B. Aazhang, "On beamforming with finite rate feedback in multiple antenna systems," IEEE Transactions on Information Theory, vol. 49, pp. 2562-2579, Oct. 2003.

[7] David J. Love,Robert W. Heath, Jr., and Thomas Strohmer, "Grassmannian beamforming for Multiple-Input Multiple-Output systems," IEEE Transactions on Information Theory, vol. 49, Oct. 2003.

[8] D. J. Love and R. W. Heath Jr. , "Limited feedback precoding for spatial multiplexing systems," in Proc. of IEEE Global Telecomm. Conf., pp. 1857-1861, Dec. 1-5 2003.

[9] D. J. Love and R. W. Heath Jr., "Limited feedback precoding for spatial multiplexing systems using linear receivers," in Proc. of IEEE Military Comm. Conf., pp. 627-632, Oct. 2003.

[10] G. Jongren and M. Skoglund, "Improving orthogonal space-time block codes by utilizing quantized feedback information," in Proc. IEEE Int. Symp. Information Theory, p. 220, June 2001.

[11] M. Skoglund and G. Jongren, "On the capacity of a multiple-antenna communication link with channel side information," IEEE J. on Selected Areas in Communications, 
vol. 21, pp. 395-405, April 2003.

[12] W. Santipach and M. L. Honig, "Asymptotic performance of mimo wireless channels with limited feedback," in Proc. IEEE Milcom, pp. 141-146, Oct. 2003.

[13] G. D. Golden, G. J. Foschini, R. A. Valenzuela, and P. W. Wolniansky, "Detection algorithm and initial laboratory results using v-blast space time communication architecture," Electron. Lett., vol. 35, pp. 1416-1418, Jan. 1999.

[14] G. Ginis and J. M. Cioffi, "On the relation between V-BLAST and the GDFE," IEEE Communications Letters, vol. 5, pp. 364-366, Sep. 2001.

[15] G. H. Golub and C. F. Van Loan, Matrix Computations. Johns Hopkins University Press, third ed., 1996.

[16] V. L. Girko, Theory of Random Determinants. Kluwer Academic Publishers, 1990.

[17] F. Hiai and D. Petz, "The Semicircle Law, Free Random Variables and Entropy," American Mathematical Society, vol. 77, 2000. Mathematical Surveys and Monographs.

[18] Allen Gersho and Robert M. Gray, Vector Quantization and Vector Compression. Kluwer Academic Publication, 1992.

[19] F. Lahouti and A. K. Khandani, "Quantization of LSF parameters using a trellis modeling," IEEE Trans. Speech and Audio Proc., vol. 11, pp. 400-412, Sept. 2003.

[20] T. R. Fischer, "A pyramid vector quantizer," IEEE Trans. Inform. Theory, vol. 32, pp. 568-583, July 1986.

[21] G. R. Lang and F. M. Longstaff, “A leech lattice modem," IEEE J. Select. Areas Commun., vol. 7, pp. 968-973, Aug. 1989.

[22] A. K. Khandani and P. Kabal , "Shaping multi-dimensional signal spaces-part ii: Shell-addressed constellations," IEEE Trans. Inform. Theory, pp. 1809-1819, Nov. 1993.

[23] J. G. Proakis, Digital Communication. McGraw-Hill, 4th ed., 2000. 